\title{
Silicon photonics in Pirelli
}

\author{
M. Romagnoli, L. Socci, L. Bolla, S. Ghidini, P. Galli, C. Rampinini, G. Mutinati, A. Nottola, A. \\ Cabas, S. Doneda, M. Di Muri, R. Morson, T. Tomasi, G. Zuliani, S. Lorenzotti, D. Chacon, S. \\ Marinoni, R. Corsini, F. Giacometti, S. Sardo, M. Gentili, G, Grasso. \\ Pirelli Labs s.p.a. viale Sarca 222, 20126, Milano, Italy
}

\begin{abstract}
Silicon is the dominant material in the microelectronic industry and silicon photonics is rapidly gaining importance as a technological platform for a wide range of applications in telecom, and optical interconnect. It allows the implementation of many photonic functions through the use of wafer-scale technologies normally used for advanced CMOS-processing. In this paper some of the most important issues toward a practical implementation of Silicon photonics into an industrial device will be addressed: low loss waveguides, polarization handling, tunability, hitless switching. A tunable Add-Drop multiplexer has been chosen as a case study of a fully integrated device.
\end{abstract}

Keywords: Silicon photonics, OADM, microring resonators, polarization splitters rotators

\section{INTRODUCTION}

In the last few years, the Internet traffic experienced a huge growth. In near future the traffic is expected to rise faster and faster driven in particular by video distribution services. In this context, the metro area of the optical network is rapidly becoming the bottleneck of the entire telecom network. Moreover there is the need to push the optical layer of the network toward the final end user: the so called optical access network. In this environment, the flexibility at the optical level becomes an important issue for metro and access optical networks. Traditional technologies for flexible optical components (like tunable lasers and Reconfigurable Add-Drop Multiplexers, OADM) are quite effective in terms of performances, but they are not in line with metro cost targets, especially for metro access application.

In order to fit in the tight metro access network requirements, consisting basically in a high level of miniaturization and lower cost, a new generation of optical components is needed, based on a new technology suitable to effectively scale costs with volumes. A very attractive candidate is silicon based integrated optics. Nanophotonics promises a dramatic scale reduction compared to contemporary photonic components. This allows the integration of many functions onto a single chip. Silicon-on-Insulator (SOI) is an ideal material for nanophotonics. Many important building blocks have already been demonstrated at the level of a proof of concept such as low loss silicon waveguides and microring filters. In this paper we review and address some important issues toward the practical implementation of SOI technology in an industrial manufacturable product. Basic building blocks like waveguide, tunable filters, polarization splitters and rotators, are also discussed as long as their integration issues.

The main topics covered in this paper consequently are: polarization handling, tunability, hitless switching. A tunable optical Add-Drop module has been chosen as an example where all the aforementioned issues have to be taken into account as a whole in view of an industrialization of such device in the near future. Nowadays optical network OADM node is composed of a discrete integration of microoptics components or a mixture of microoptics and integrated optics basic functionalities as individual filters, splitters, or simple switches that form a layout reminiscent of the electronic systems of the 60 s, i.e. before the introduction of integrated circuits.

Flexibility and reconfigurability are key features to win the battle for the next generation optical networks. These functions can be achieved through the intrinsic high tunability of silicon devices. However, in spite of its large thermooptic effect, tuning a single filter over the entire $\mathrm{C}$-band of the telecommunications or even more $(\mathrm{C}+\mathrm{L}$ bands), remains a challenging task. High temperature is needed and too high power consumption is necessary, especially when the small volume of the packaging is taken into account.

The solution of the problem of extended tunability is twofold: on one side the Vernier approach has been adopted, on the other side filter architecture has been chosen in order to ensure the Vernier spurious peaks do not affect the propagating signals both during the filter operation and during the switching from one channel to the other.

Silicon Photonics and Photonic Integrated Circuits, edited by Giancarlo C. Righini, Seppo K. Honkanen, Lorenzo Pavesi, Laurent Vivien, Proc. of SPIE Vol. 6996, 699611, (2008) · 0277-786X/08/\$18 · doi: 10.1117/12.786539 
Polarization transparent operation has been achieved by the use of the polarization diversity scheme depicted in Fig. 5a). In order to make an efficient use of the scheme described in Fig. 5a) an integrated Polarization Splitter and Rotator (PSR) is needed. This important building block has been theoretically investigated and practically demonstrated in SOI.

In the following chapters all these basic building blocks are discussed.

\section{SOI BASIC BUILDING BLOCKS}

\subsection{Waveguide}

Silicon wire waveguides have two very important advantages over other waveguides. First of all, they provide a very high confinement of light, which is necessary to build microring resonators with small radius of curvature and hence large Free Spectral Range (FSR). On the other hand, Silicon has a very high thermo-optical coefficient that enables a large tunability.

Conversely, they have some disadvantages. The first is that their tiny dimensions lead to high polarization sensitivity; the second is that they suffer from scattering losses, mainly due to sidewall roughness. The first issue can be solved by applying a polarization diversity scheme which involves a Polarization Splitter and Rotator (PSR) as the one described in Fig. 5b). A proper choice of the geometric parameters of the waveguide can alleviate the second one, as shown later.
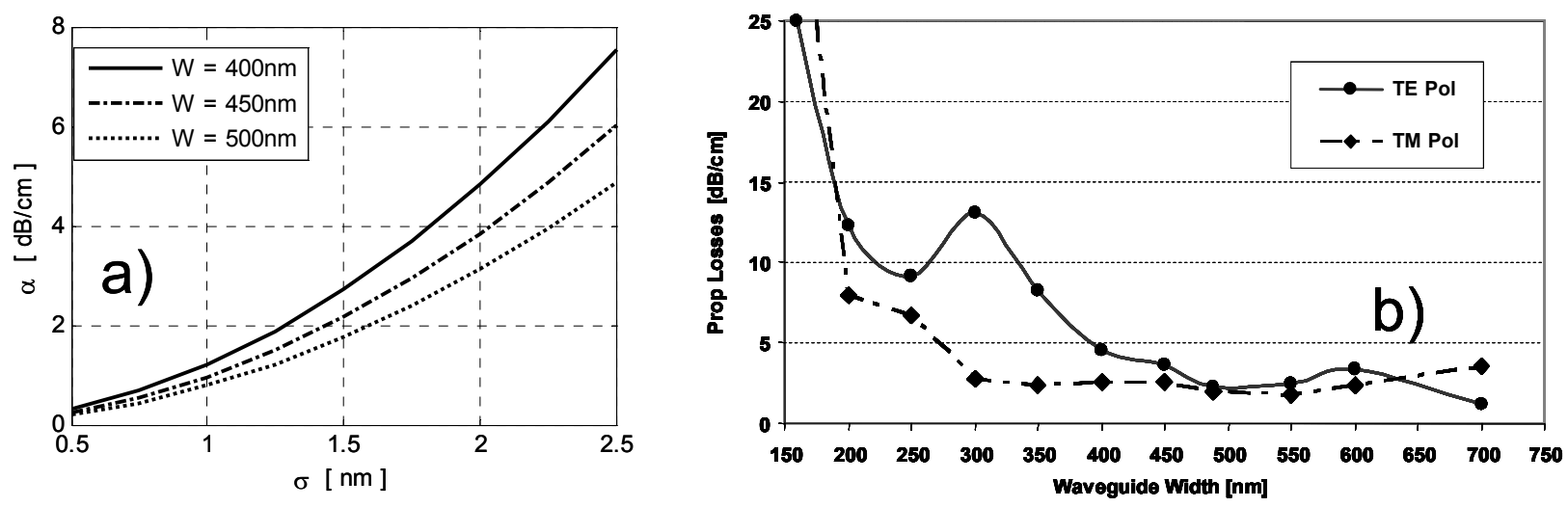

Fig. 1). On the left hand side, simulated scattering losses for three $220 \mathrm{~nm}$ high buried SOI waveguides for TE polarization; on the right hand side, experimental propagation losses of $220 \mathrm{~nm}$ high buried SOI waveguides for TE and TM polarization.

Pirelli [1] has developed a suitable silicon wire waveguide to tackle all these problems, with a height of $220 \mathrm{~nm}$. Single mode operation fixes an upper bound to its width of about $550 \mathrm{~nm}$ and technological process fixes a lower bound of about $100 \mathrm{~nm}$. Fig. 1a) shows a numerical computation of scattering losses [1] versus roughness for three different waveguide widths: it is clear that wider waveguides suffer from smaller scattering losses. This result also shows the extremely strong dependence of propagation loss on the amount of surface roughness, and indicates the nanometric control required to achieve a waveguide fabrication suitable for application. A confirmation comes from the experiment shown in Fig. 1b), which shows a maximum of the propagation losses for a width of $300 \mathrm{~nm}$. Below $300 \mathrm{~nm}$, leakage in the substrate becomes dominant. In the widths range between 400 and $490 \mathrm{~nm}$, the propagation losses obtained are $<3 \mathrm{~dB}$ for $\mathrm{TE}$ and $<2 \mathrm{~dB}$ for TM.

In terms of tunability, considering a microring filter with a $2300 \mathrm{GHz} F \mathrm{FR}$, the tunability provided by such a waveguide is $77 \mathrm{pm} /{ }^{\circ} \mathrm{C}$.

\subsection{T-OADM filter description}

Tunable Add-Drop multiplexer has been chosen as a case study where all the aforementioned building blocks have to be integrated together. The first important functionality that exploits the material properties in terms of large index of refraction contrast and sensitivity to temperature is the microring filtering. As mentioned earlier, tunability over an extended $\mathrm{C}$-band (or $\mathrm{C}+\mathrm{L}$ bands) requires a twofold solution: on one side a Vernier approach, on the other side a 
particular filter architecture has been chosen in order to ensure that the Vernier spurious peaks do not affect the propagating signals both during the filter operation and during the switching from one channel to the other. More precisely, this requirement is also called "hitless switching", defined as the capability of the filter to tune from one channel to the other without affecting the Bit Error Rate of the transmission system. Finally, we seek for a filter with $>30 \mathrm{~dB}$ of dropped channels rejection, $>30 \mathrm{~dB}$ of adjacent channels isolation and $25 \mathrm{GHz}$ of bandwidth at $0.5 \mathrm{~dB}$.

We present a filter composed by a double-stage second-order microring Vernier resonators. Fig. 2a) schematically represents the complete filter, as composed by 3 second-order filters. Let's call filter $A$ the left-most side second-order filter, filter $B$ the right-most one on the thru arm and filter $C$ the bottom-most one on the drop arm. Filter $A$ and filter $B$ use the Vernier effect, while filter $C$ doesn't.

The first filter's parameters are chosen so that, by exploiting the Vernier effect, the effective FSR is greater than 4300 $\mathrm{GHz}$ so that only one active resonance is present in the whole $4 \mathrm{THz}$ wide $\mathrm{C}$-band.

The second filter, filter $B$, is needed to meet the specifications of the thru port while the third filter, filter $C$, is necessary to meet the specifications of the drop port. In this case the two rings are equal.

Fig. 2b) and Fig. 2c) show respectively the theoretical amplitude spectrum and dispersion of the complete filter of Fig. 2a) both in through and drop, overlapped to the black rectangle representing a DWDM channel. The required specifications previously listed are all met for both trough and drop ports.
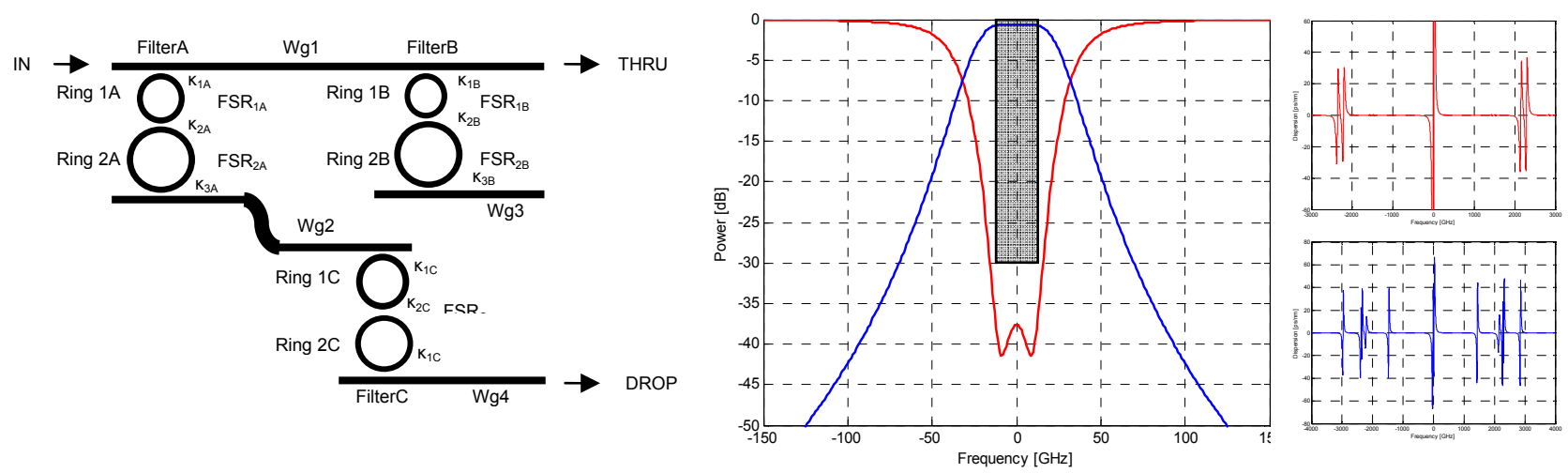

Fig. 2). a) Schematics of a double stage filter; b) Theoretical spectrum of the complete filter (drop in blue, thru in red, channel's requirements in black); c) dispersion on drop port in red and on thru in blue.

A realization of the complete double stage filter is shown in Fig. 3.c), for the aforementioned waveguide of $220 \mathrm{~nm}$ height. Coupled mode theory and FDTD simulations have been used to compute the physical parameters of the filters, such as radiuses of curvature and gaps. In Fig. 3.a) a SEM image of the coupler between a straight waveguide and a ring it is shown.

It must be noted that for this type of feature sizes the dimensional tolerances are of the order of nanometers. For instance, the variation of waveguide width of $1 \mathrm{~nm}$ leads to a shift of the resonant frequency of about $100 \mathrm{GHz}$, and analogously for a variation of the thickness. Individual ring trimming is therefore required to obtain and control the spectral alignment of resonances as it is shown in Fig. 3b).

Fig. 3.c) is a close view of the spectrum after the two stages in the through port and only one stage in the drop one. A broader look over the whole C-band would reveal spurious peaks on the drop signal, though: these are clearly visible in the bottom picture at the left hand side of Fig. 4a), for a single-stage filter. The spurious peaks of the single stage are around $20 \mathrm{~dB}$ below the drop maximum.

We also report the demonstration of the tunability of the single-stage double-ring Vernier microring resonator, shown in Fig. 2a) as filter $A$. 

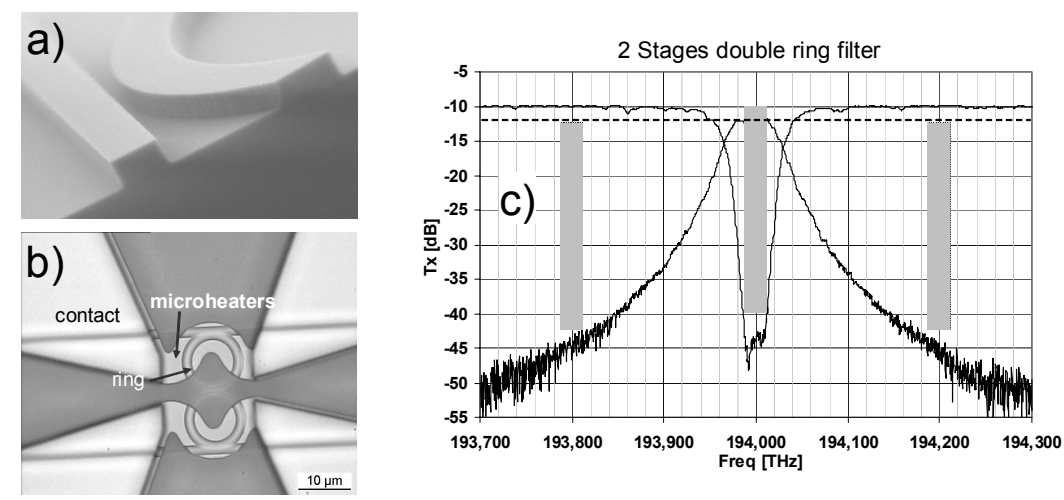

Fig. 3). a) SEM image of the ring/bus waveguide cross section before deposition of the top cladding, b) optical microscope image of a single stage as appears after deposition of top cladding and microheaters with contact microstripes, c) through and drop spectral response of a two stage double ring filter cascade.

Tuning of filter $A$ is carried out in three consecutive steps.

1. First of all, the filter is disabled by detuning the resonant frequency of the two rings; in this "disabled" state the whole filter behaves like an all-pass one and its response is flat over the whole C-band, except for little notches due to coupling and propagating losses in the ring.

2. Then, both the first and the second ring are tuned to the destination channel. If the coupling and propagation losses in the ring are negligible, perturbation of the input signals is small, hence achieving hitless tuning.

3. The filter is enabled back by tuning again the resonant frequency of the first ring to match the one of the second.

Fig. 4) shows the thermal tuning of 9 channels in the C-band, for a total of a $4 \mathrm{THz}$ tuning. By using the nearest resonance of each microring and the Vernier effect, we can achieve such a broad tuning range with a power as little as 80 $\mathrm{mW}$ (42 $\mathrm{mW}$ for the bigger ring and 38 for the smaller one). It is interesting to note that the filter's shape and spectral features are preserved upon all the extent of tuning.
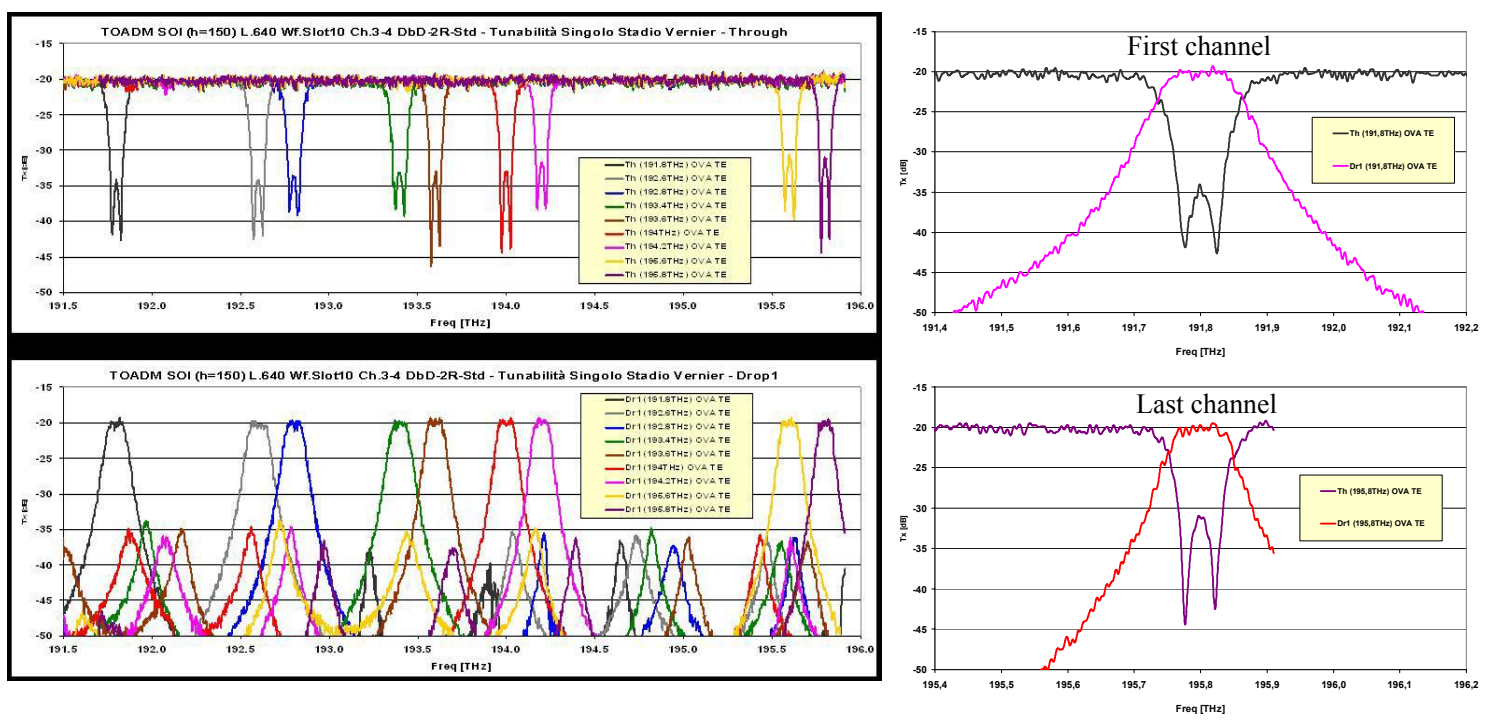

Fig. 4).Tuning of the single stage Vernier filter. On the right hand side, a zoom of the first and last channels of the C-band: the shape of the filter is conserved upon tuning. 


\subsection{Polarization}

Polarization transparency is one of the trickiest issues to be addressed in view of a practical implementation of the potentiality of the silicon photonics. The waveguides and filters described so far, exhibit strong polarization behavior; specifically they are designed and optimized to deal with the TE polarization state. On the other hand the polarization state coming from the transmission line is random and varies both with time and with wavelength. Two main approaches have been suggested in the past to overcome this problem. The first possibility is to design polarization independent waveguides. Due to the extremely high index contrast between the Silicon core and the Silicon Oxide cladding ( $\Delta \mathrm{n}>$ $40 \%$, this is a very challenging task [4][5][6][7][8][9][10] and impose several constrains on the guiding properties of the waveguides which hinder the realization of more complex functionalities. It has been shown [1][2][12][13][14]Error! Reference source not found. that rather than pursuing a problem of making polarization insensitive the entire filter, a polarization diversity scheme may be conveniently exploited to overcome the problem. A polarization diversity scheme is shown in Fig. 5a).

Polarization diversity relies first on splitting the incoming polarization in the TE and TM components and separate in two arms and then TM rotated into TE. The filter is then designed only for TE polarization and duplicated in two separated arms. The output of the scheme, analogously to the input, consists in a rotation and recombination of the two different polarizations in one output fiber. The scheme described in Fig. 5a) it is not an interferometric one and does not give rise to intensity modulation of the outgoing signal.

The polarization diversity scheme of Fig. 5a) can be implemented at different levels of integration. On one hand it is possible to manage the polarization state of the incoming light externally from the silicon chip by a combination of microoptics polarization splitters and waveplates. This approach works in principle but has the strong disadvantage of severely limiting the integration and increasing the overall cost and footprint of the final module.

Chip integrated Polarization Splitters (PS) and Rotators (PR) are both needed to fully exploit the monolithic integration possibility offered by the SOI platform. In this way would be possible to use a front end wafer level fabrication process leading to a great simplification of packaging and cost reduction.

A chip integrated Polarization Splitter-Rotator (PSR) based on adiabatic modes evolution has been originally conceived by M. Watts et al. [1] [14] and successfully demonstrated at a level of proof of concept in silicon nitride platform by an MIT group [12]. Here we describe a device based on basically the same approach and we demonstrate how that concept works and is rescaled to the SOI platform where the index contrast is much higher than silicon nitride.

The adiabatic mode evolution approach has been preferred to a more classic mode coupling scheme, i.e. integrated waveplates and retarders [11], because it exhibits a high optical bandwidth and more important is intrinsically more tolerant to fabrication errors than any "non adiabatic" approach. The last point is particularly important in the extremely high index contrast environment typical of the SOI technological platform.

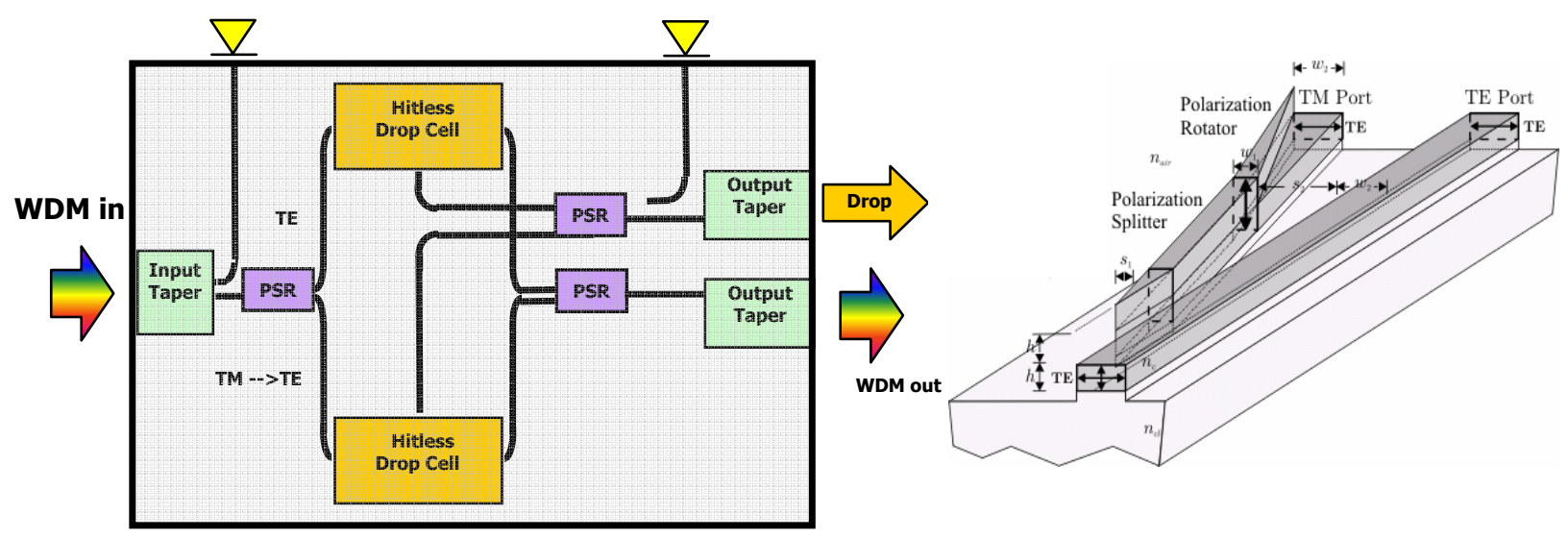

Fig. 5). a) Polarization diversity scheme b) Polarization Splitter and Rotator: longitudinal view and cross sections. 
A pictorial view of the entire PSR is depicted in Fig. 5b). The PSR is intrinsically a two lithographic layer device. If only polarization splitting was a concern it would be possible to use only one lithographic layer; polarization rotation on the other hand needs a breaking of symmetry in two planes. This could be done by the use of a slanted etching process as suggested in [3][4] but for technological reasons we preferred to use a two layers approach. Reference is now made to Fig. 5b) where the pictorial view of the SOI-PSR is shown. The PS is basically constituted by one horizontal and one vertical waveguides brought to a close proximity. The horizontal waveguide is the standard 450 by $220 \mathrm{~nm}$ silicon waveguide. By inspecting the modal field distribution of the end section of the PS it is clear how that structure supports four guided modes in the following order:

1. Mode 1, TE, mainly confined in the horizontal waveguide;

2. Mode 2, TM, mainly confined in the vertical waveguide;

3. Mode 3, TM, mainly confined in the horizontal waveguide;

4. Mode 4, TE, mainly confined in the vertical waveguide.

By tapering the vertical waveguide down, as at the beginning of the PS in Fig. 5b), modes 3-4 will be unguided and modes 1-2 will become respectively the TE and TM modes of the horizontal waveguide. This means that the device will behave as a polarization splitter provided that mode coupling is prevented, i.e. if the structure is long enough to be considered adiabatic.

In this case in fact the first mode at the beginning of the propagation (TE of the horizontal waveguide) will adiabatically evolve in the first mode of the end section: the TE mainly in the horizontal waveguide. The same will apply to the TM mode at the beginning of the PS which will evolve into the mode 2 of the final end of the PS with all the power basically confined in the vertical waveguide. Modes 3-4 will never be excited if the device is adiabatic. In practice some mode coupling happens and some power could end in the unwanted modes. This constitutes a cross talk which can be kept to a level below $-20 \mathrm{~dB}$ by a proper choice of the design parameters of the PSR like: waveguide widths and heights, gap and taper profiling.

After the modes have been split, the horizontal and the vertical waveguides can be separated until they do not interact anymore. After this separation step, the polarization rotator starts in the vertical waveguide, while the horizontal one continues unperturbed, because it already guides a TE polarized light.

The PR will rotate the polarization of the light in the vertical waveguide from TM to TE, and interface a "conventional" horizontal waveguide. Again, the polarization rotation is based on the adiabatic principle and mode evolution. The geometry of the vertical waveguide is slowly changed along the longitudinal direction, reducing the width of the upper layer and increasing the width of the underlying one. At the end of the PR, the cross section of the waveguide is very similar to a horizontal waveguide, i.e. a Silicon channel waveguide of $450 \mathrm{~nm}$ width, with a small tip on top of it. Those particular tapering rules of the top and bottom layers ensure the breaking of symmetry required for the PR to properly operate. In other words, the shape variation of the vertical waveguide in the PR is the optical analogous of twisting the vertical waveguide itself to a horizontal one. The interface with the output horizontal waveguide has two consequences: losses, due to reflection and to radiation, and crosstalk, due to excitation of both the guided modes (TE and TM) of the output waveguide. Again this crosstalk can be minimized by a suitable choice of the PR design parameters like: waveguide and tip width and tapering rules. Simulations show negligible losses and a crosstalk below $-20 \mathrm{~dB}$.

Experimental performances of the fabricated PSR are now discussed starting with the PSR losses which are defined as the extra losses induce by the PSR with respect to a straight horizontal waveguide (450 X $220 \mathrm{~nm}$ ) of the same length; they are lower than $0.5 \mathrm{~dB}$ for the TM polarization and negligible $(<0.1 \mathrm{~dB})$ for the TE polarization. This result is very reproducible and it is based on statistical measurements taken on many chips of several wafers.

Reference is now made to Fig. 6a) where the measured PSR performances are reported versus length for TE and TM input respectively at the wavelength of $1545 \mathrm{~nm}$. Three cross talks can be defined in a very straightforward fashion with reference to Fig. 5b) for each input polarization state. Powers in both TE and TM polarizations are measured at both TE and TM output ports. In the case of TE launch most of the power is output at TE port as TE light and its value is used as the normalization. The first cross talk is then defined as the residual TE power in the TM port. This is in practice a measurement of the PS efficiency. The second and third cross talks are then the TM power residual in the TE and TM port respectively. 
The same holds when launching the TM mode. In this case most of the power is output at TM port as TE light and it is used as the reference level for the normalization. The first cross talk in this case is defined as the residual TM light in the output TM port which is in practice a measurement of the PR efficiency. The second and third cross talks are respectively the TE and the TM powers residual in the TE output port, these quantities are connected to the PS efficiency.

As stated by the adiabatic principle, all the defined cross talk monotonically decrease with increasing the PSR length being lower than $-20 \mathrm{~dB}$ at a length of about $350 \mu \mathrm{m}$ for any input polarization state
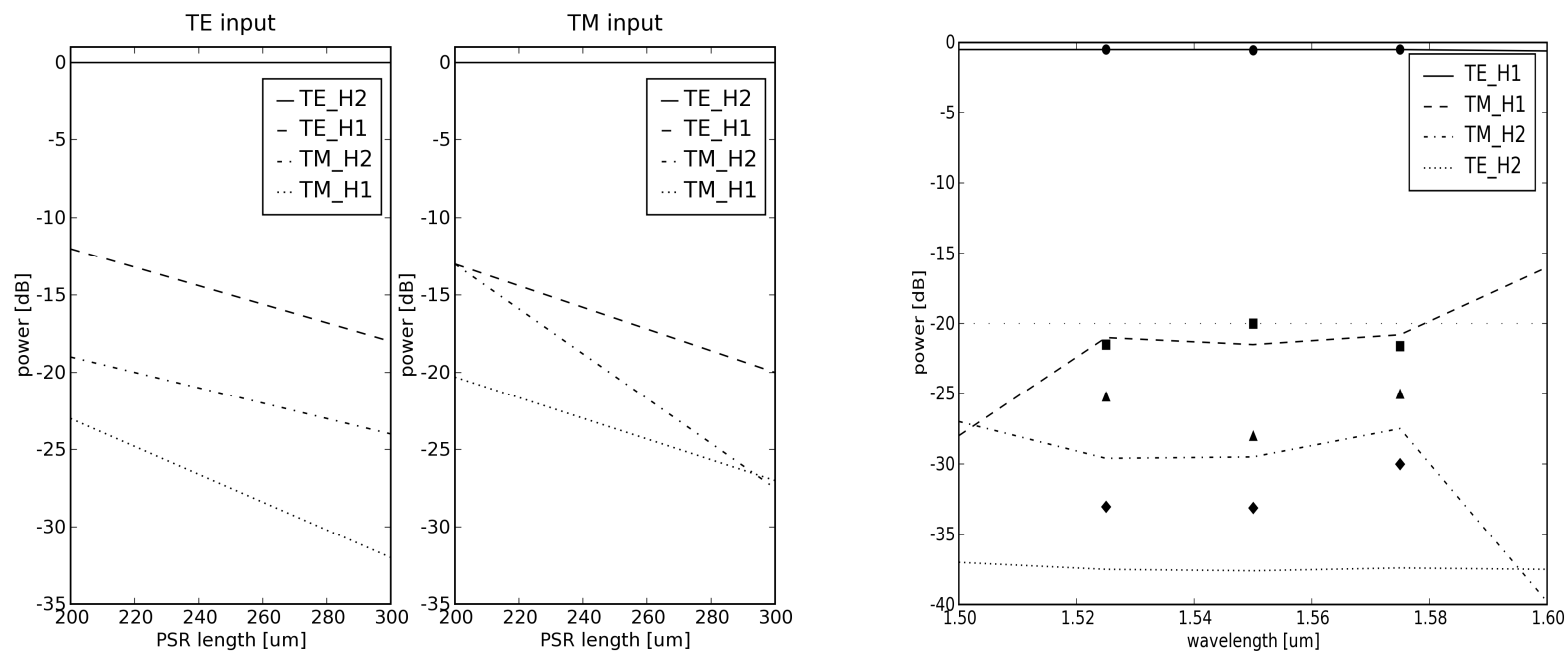

Fig. 6). PSR crosstalks at wavelength $1545 \mathrm{~nm}$. Performances improve as the PSR length increases. Experimental PSR performances over the whole C-band, for TM input, which is more critical than the TE input. Agreement between simulated values (lines in graphs) and experimental values (symbols) is remarkable. Crosstalks are below -20dB over the whole C-band.

In Fig. 6b) the PSR performances versus wavelength are reported for a PSR length of $350 \mu \mathrm{m}$. A comparison between the measured performances of the fabricated PSR (symbols) and the simulated ones (lines in graph) show very good agreement.

The case reported in Fig. 6b) refers to an input TM polarization, which is the trickiest one, because it's the one traveling along the complete PSR (splitter + rotation). It is evident that both the losses and the crosstalks experienced from the TM polarization from going through the entire device are quite flat with respect to wavelength and basically constant over the C-band. Not shown, even better results are obtained for a TE input, as expected from theory.

\section{CONCLUSIONS}

In this paper we reviewed the main advantages of the combination of the silicon material and SOI properties along with the possibility of achieving an ultra compact, low cost, multi-functionality integrated structure. As a result we have successfully demonstrated a low power consumption $4 \mathrm{THz}$ hitless tuning of an SOI based T-OADM functionality for metro network core/access application.

Polarization transparent operation has also been achieved through the use of integrated Polarization Splitter and Rotator in SOI technology with losses below $0.5 \mathrm{~dB}$ and crosstalk lower than $-20 \mathrm{~dB}$ over the C-band for both the output ports. 


\section{REFERENCES}

[1] Cabas, A.; Di Muri, M.; Doneda, S.; Galli, P.; Ghidini, S.; Giacometti, F.; Lorenzotti, S.; Mutinati, G.; Nottola, A.; Romagnoli, M.; Sardo, S.; Socci, L.; Tomasi, T.; Zuliani, G.; Gentili, M.; Grasso, G.; "Silicon on Insulator Based Integrated Tunable Add \& Drop Filter for Metro DWDM Networks", Transparent Optical Networks, 2007. ICTON '07. 9th International Conference on, Vol 1, 2007, p.236-239

[2] M.R. Watts, M. Qi, T. Barwicz, L. Socci, P.T. Rakich, E.P. Ippen, H.I. Smith, H.A. Haus, "Towards integrated polarization diversity: design, fabrication and characterization of integrated polarization splitters and rotators," in Opt. Fiber Comm. Conf. Tech. Digest, Vol. 6, 2005, p31

[3] H. El-Refaei, D. Yevick, and T. Jones, "Slanted-Rib Waveguide InGaAsP-InP Polarization Converters," J. Lightwave Technol. 22, 1352- (2004)

[4] Henghua Deng; Yevick, D.O.; Brooks, C.; Jessop, P.E.; "Design rules for slanted-angle polarization rotators", J. Lightwave Technol. 23(1), 432 (2005)

[5] Qing Guo, Wei-Ping Huang, $\mathrm{Chi} \mathrm{Wu}$, “A polarization-independent coupled-waveguide grating resonator filter", Conference Proceedings. LEOS '96 9th Annual Meeting. IEEE Lasers and Electro-Optics Society 1996 Annual Meeting (Cat. No.96CH35895), 1996, p 309-10 vol.2

[6] Amersfoort, M.R; Soole, J.B.D.; LeBlanc, H.P.; Andreadakis, N.C.; Rajhel, A.; Caneau, C.; Koza, M.A.; Bhat, R.; Youtsey, C.; Adesida, I.; "Polarization-independent InP-arrayed waveguide filter using square cross-section waveguides", OFC'96. Optical Fiber Communication. Vol.2. 1996 Technical Digest Series. Conference Edition (IEEE Cat. No.96CH35901), 1996, p 101-2

[7] Bergstein, L.; "Polarization-independent optical waveguide", Topical meeting on integrated optics-guided waves, materials, and devices, 1972, p MB5 1 pp.

[8] Cassan, E.; Vivien, L.; Laval, S. Source; "90deg-turns with polarization-independent single-mode waveguide on silicon-on-insulator for telecommunication wavelengths", 11th European Conference on Integrated Optics. Proceedings, March 2003, p 225-8 vol.1

[9] Seong Phun Chan; Ching Eng Png; Soon Thor Lim; Reed, G.T.; Passaro, V.M.N.; "Single-mode and polarizationindependent silicon-on-insulator waveguides with small cross section", Journal of Lightwave Technology, v 23, n 6, June 2005, p 2103-11

[10] Worhoff, K.; Roeloffzen, C.G.H.; de Ridder, R.M.; Sengo, G.; Hilderink, L.T.H.; Driessen, A.; "Tolerance of polarization independent waveguides for communication devices", Proceedings of the SPIE - The International Society for Optical Engineering, v 5451, n 1, 2004, p 369-80

[11] Shintaku, T.; Sugimoto, N.; Shimokozono, M.; Tate, A.; Kubota, E.; Kozawaguchi, H.; Ishii, M.; Inoue, Y.; "Waveguide polarization-independent optical circulator using a Mach-Zehnder interferometer", 11th International Conference on Integrated Optics and Optical Fibre Communications 23rd European Conference on Optical Communications. IOOC-ECEC97. Post Deadline Papers (Conf. Publ. No.448), 1997, p 57-60 vol.5

[12] Watts, M.R.; Minghao Qi; Barwicz, T.; Socci, L.; Rakich, P.T.; Ippen, E.P.; Smith, H.I.; Hermann, A.H.; “Towards integrated polarization diversity: design, fabrication, and characterization of integrated polarization splitters and rotators", 2005 Optical Fiber Communications Conference Post deadline Papers (IEEE Cat. No. 05CH37672), 2005, p 3 pp. Vol. 5

[13] Watts, M.R.; Haus, H.A.; Ippen, E.P.; "Integrated mode-evolution-based polarization splitter", Optics Letters, v 30 , n 9, 1 May 2005, p 967-9

[14] Watts, M.R.; Haus, H.A.; "Integrated mode-evolution-based polarization rotators", Optics Letters, v 30, n 2, 15 Jan. 2005, p 138-40

[15] Watts, R.A.; Sambles, J.R.; "Polarization conversion from blazed diffraction gratings", Journal of Modern Optics, v 44, n 6, June 1997, p 1231-41

[16] Tzolov, V.P.; Fontaine, M.; "A passive polarization converter free of longitudinally-periodic structure”, Optics Communications, v 127, n 1-3, 1 June 1996, p 7-13 